\title{
edoc
}

Institutional Repository of the University of Basel

University Library

Schoenbeinstrasse 18-20

CH-4056 Basel, Switzerland

http://edoc.unibas.ch/

Year: 2012

\section{Integrated diamond networks for quantum nanophotonics}

Hausmann, Birgit J. M. and Shields, Brendan and Quan, Qimin and Maletinsky, Patrick and McCutcheon, Murray and Choy, Jennifer T. and Babinec, Tom M. and Kubanek, Alexander and Yacoby, Amir and Lukin, Mikhail D. and Lončar, Marko

Posted at edoc, University of Basel

Official URL: http://edoc.unibas.ch/dok/A6337984

Originally published as:

Hausmann, Birgit J. M. and Shields, Brendan and Quan, Qimin and Maletinsky, Patrick and McCutcheon, Murray and Choy, Jennifer T. and Babinec, Tom M. and Kubanek, Alexander and Yacoby, Amir and Lukin, Mikhail D. and Lončar, Marko. (2012) Integrated diamond networks for quantum nanophotonics. Nano Letters, Vol. 12, H. 3. S. 1578-1582.

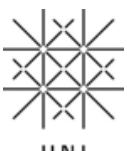




\title{
Integrated diamond networks for quantum nanophotonics
}

\author{
Birgit J.M. Hausmann ${ }^{1}$, Brendan Shields², Qimin Quan ${ }^{1}$, Patrick Maletinsky², Murray McCutcheon ${ }^{1}$, Jennifer T. \\ Choy $^{1}$, Tom M. Babinec ${ }^{1}$, Alexander Kubanek ${ }^{2}$, Amir Yacoby ${ }^{2}$, Mikhail D. Lukin ${ }^{2}$, Marko Lončar ${ }^{1 *}$ \\ 1. Harvard University, School of Engineering and Applied Sciences, 33 Oxford Street, Cambridge, USA and \\ 2. Harvard University, Department of Physics, 17 Oxford Street, Cambridge, USA
}

Diamond is a unique material with exceptional physical and chemical properties that offers potential for the realization of high-performance devices with novel functionalities. For example diamond's high refractive index, transparency over wide wavelength range, and large Raman gain are of interest for the implementation of novel photonic devices ${ }^{1}$. Recently, atom-like impurities in diamond emerged as an exceptional system for quantum information processing $2+4$, quantum sensing $5-7-7$ and quantum networks ${ }^{8 \mid 9}$. For these and other applications, it is essential to develop an integrated nanophotonic platform based on diamond. Here, we report on the realization of such an integrated diamond photonic platform, diamond on insulator (DOI), consisting of a thin single crystal diamond film on top of an insulating silicon dioxide/silicon substrate. Using this approach, we demonstrate diamond ring resonators that operate in a wide wavelength range, including the visible $(630 \mathrm{~nm})$ and near-infrared $(1,550 \mathbf{~ n m})$. Finally, we demonstrate an integrated, on-chip quantum nanophotonic network, consisting of ring resonators coupled to low loss waveguides with grating couplers, that enables the generation and efficient routing of single photons at room temperature.

With its large bandgap $(5.5 \mathrm{eV})$ and negligible nonlinear loss mechanisms due to multi-photon processes at visible (VIS) and near-infrared (NIR) wavelengths 10 , diamond is an ideal host material for optical devices. However, until recently diamond photonics has been limited to bulk $k^{11}\left[16\right.$ or polycrystalline diamond devices ${ }^{17}[19]$ due to difficulties associated with the fabrication of thin, single crystal, diamond (SCD) films on sacrificial or low index substrates. Light absorption and scattering at grain boundaries can be detrimental for the polycrystalline diamond approaches, while the realization of scalable, on-chip quantum networks can be difficult with single-crystal bulk diamond approaches.

Our approach involves the fabrication of high quality, low loss ring resonators and simple quantum photonic networks directly in SCD thin slabs. Figure 1 1 illustrates our fabrication sequence, based on the approach that we 2021 as well as other ${ }^{22}$ have recently demonstrated. First we thin a $20 \mu \mathrm{m}$ thick type Ib single crystal diamond slab (Element Six) to the preferred device layer thickness by an oxygen-based inductively coupled reactive ion etch (ICP RIE) ${ }^{7}$. An e-beam (Elionix) exposes XR e-beam resist (spin-on-glass, Dow Corning) to form a mask which we transfer to the diamond film in a second etch. Figure 1 b shows a scanning electron microscope (SEM) image of representative diamond ring resonators on $\mathrm{SiO}_{2} / \mathrm{Si}$ substrate.

To explore the broadband nature of diamond photonics, we characterize the diamond ring resonators in the NIR and VIS wavelength range (Fig. 2a, see Methods). The devices, designed for single mode operation at telecom wavelengths, have an outer ring radius of $20 \mu \mathrm{m}$ and a $1 \mu \mathrm{m} \times 410 \mathrm{~nm}$ cross-section with $300 \mathrm{~nm}$ XR covering the diamond. For characterization in NIR, we probe the devices using a tapered fiber ${ }^{23}$. Figure $2 \mathrm{~b}$ shows a representative normalized transmission spectrum, which features regularly spaced dips that correspond to optical resonances of the structure. We extract a quality factor of $Q \approx 9,000$ at $\lambda=1,487.4 \mathrm{~nm}$, corresponding to a finesse of $F \approx 100$. Both quantities decrease significantly for longer wavelengths (i.e. $Q \approx 700, F \approx 12$ for $\lambda=1,676 \mathrm{~nm}$ ), which we attribute to a larger scattering loss at the outer ring edge caused by reduced confinement in the high index material $\left(n_{\text {diam }}=2.4\right)$. Furthermore, we perform measurements in the over-coupled limit where the field decay rate to the tapered fiber, $\tau_{\mathrm{ex}}^{-1}$, exceeds the intrinsic loss rate of the ring resonator, $\tau_{0}^{-1}$. This introduces a fast decay channel of the resonator field for efficient routing of the photons into the desired collection channel, but at the same time reduces the overall quality factor of the resonator. This effect is more dramatic at long wavelengths. On resonance the transmission drops by $18 \%$. A perturbation of the phase of the transmitted wave, e.g. due to partially reflecting elements in the fiber ${ }^{24}$, leads to a complex interference behavior. The lineshape of the cavity resonance therefore changes from a Lorentzian to Fano-like shape, expressed by the so-called Fano parameter $\zeta$ ( $\zeta=0$ for a Lorentzian lineshape). We describe the normalized transmitted intensity $\mathrm{T}$, by

$$
T(\Delta)=1-\frac{4 \xi(1-\xi)(\kappa / 2+\zeta \cdot \Delta)^{2}}{(\kappa / 2)^{2}+\Delta^{2}\left(1+\zeta^{2}\right)}
$$

Here $\kappa$ is the cavity linewidth (FWHM) and $\Delta$ is the detuning of the probe frequency from the resonance frequency. $\xi$ is the coupling parameter described by $\frac{\tau_{0}}{\tau_{0}+\tau_{\mathrm{ex}}}$. We fit our spectrum to a value of $\xi \approx 0.95$ with $\zeta \approx 0.33$ for a wavelength of $1487.4 \mathrm{~nm}$, resulting in a coupling parameter, $\eta_{\mathrm{ex}}=18$, given by

$$
\eta_{\mathrm{ex}}=\left(\frac{1}{\xi}-1\right)^{-1}
$$


Therefore, the coupling rate to the tapered fiber is roughly 18 times faster than the intrinsic loss rate, which allows us to estimate the intrinsic quality factor of our resonator to be $Q_{\mathrm{i}}=1.6 \cdot 10^{5}$.

To illustrate the broadband nature of diamond photonics we characterize the same ring resonator in the VIS wavelength range, using the fluorescence of diamond impurities. We use a photoluminescence approach in a twocollection arm scanning confocal microscope $\mathrm{e}^{25}$ (Fig. 2 and Methods). Green pump light $(532 \mathrm{~nm})$ excites the devices (mounted on a 3D scanning stage) at normal incidence (Fig. 2k), and red photons $(650 \mathrm{~nm}-800 \mathrm{~nm})$ emitted from Nitrogen Vacancy (NV) color centers are collected and analyzed after passing a dichroic mirror (DM) and several filters. Our detection path is split into two arms, one of which is always fixed at the excitation spot (C1) while the second arm can be scanned independently (C2) which allows us to spatially separate excitation and collection of the system. First, we scan the sample stage to obtain an emission image of the device using C1. Figure 2 (right), shows a scan of the photon collection position over the ring in $\mathrm{C} 2$ (yellow circle) while constantly exciting with the pump laser in the same position (red circle). The intensity profile of the ring indicates excitation of a higher order mode (confirmed by 3D finite difference time domain (FDTD) simulations, not shown). The spectrum reveals multimode behavior of the cavity with $\mathrm{Q}$-factors of $\mathrm{Q} \approx 12,600$ and a finesse of 62 (Fig. $2 \mathrm{~d}$ and inset).

To further improve device functionality, we fabricate ring resonators designed to operate in a single-mode regime in the VIS wavelength range. Furthermore, we monolithically fabricate optical waveguides to provide efficient and robust in- and out-coupling of light to the resonator. The waveguides contain second order gratings on each end to facilitate free-space coupling of photons (Fig. 3a). We characterize the structure by coupling the light from a broadband white light source into one grating and by collecting transmitted light from the other grating. The transmission spectrum shows regularly spaced dips corresponding to the different (longitudinal) resonant modes of the ring resonator (Fig. $3 \mathrm{~b}$ ). Using the same approach as above, we estimate $\eta_{\mathrm{ex}} \approx 3.4, \mathrm{Q} \approx 2500$ and $\mathrm{F} \approx 40$ for the resonance at $\lambda=689.8 \mathrm{~nm}$. Here, we operate closer to critical coupling where the decay rate to the waveguide would equal the intrinsic field decay rate of the resonator $\left(\eta_{\mathrm{ex}}=1\right)$. In addition, the lineshape is Lorentzian $(\zeta \approx 0.05)$.

Finally, we demonstrate the application of our platform as an integrated quantum network ${ }^{4126}$. Specifically, we demonstrate efficient generation and routing of nonclassical light fields provided by a single quantum emitter, an NV center embedded inside our diamond waveguide-coupled ring resonator, at room temperature. Photons emitted from the NV center into the ring resonator couple evanescently to the waveguide and are outcoupled one by one at the gratings. Figure $4 \mathrm{a}$ ) and b) illustrate scans using the two-arm confocal collection channels $\mathrm{C} 1$ and $\mathrm{C} 2$, respectively (the indicated device is not the same as above (Fig. 3)). We excite an NV center with green light (532 nm) and $C_{k i}$ denotes fluorescence collected at position $i(i=\mathrm{NV}$ position (1) or waveguide ends (2,3)) by two different collection arms, $k$ $(k=1,2)$. We measure in Hanbury Brown and Twiss $(\mathrm{HBT})$ configuration $^{27}$ to evaluate the second-order intensity correlations $g^{(2)}(\tau)$ where nonclassical light behavior from a single quantum emitter results in $g^{(2)}(0)<0.5^{28}$. First we study the free-space emission of the NV center (Fig. 4h). Here, light is directly emitted upwards and extracted at the pump position in each collection position $\left(C_{11}\right.$ and $\left.C_{21}\right)$. The autocorrelation between $C_{11}$ and $C_{21}$ shows strong photon antibunching demonstrating the single photon character of the emitted quantum field. The increased coincidence rate for $12 \mathrm{~ns}<\tau<550 \mathrm{~ns}$ is attributed to an intermediate shelving state, characteristic of an NV center's emission 29 . When collecting photons emitted directly above the NV center (combining $C_{11}$ and $C_{21}$ ) we observe the typical NV center's emission spectrum (Fig. $4 \mathrm{~s}$ ) where the majority of collected photons are emitted directly into the free-space without coupling into the ring modes. Furthermore, the Raman line occurs at the same spectral position $(573 \mathrm{~nm})$ as in bulk diamond, indicating a good film quality (Fig. 4. as denoted by $\mathrm{R}$ in all spectra). The emitted spectra at the outcoupling gratings (Fig. 4f and 4 4 ) feature prominent peaks indicating coupling of the NV center's fluorescence to the modes of the ring resonator as well as transfer of emitted photons into the waveguide. Based on this fluorescence spectrum we measure Q-factors as high as $(3.2 \pm 0.4) \cdot 10^{3}$ at $665.9 \mathrm{~nm}$. Moreover, we observe the evidence of routing of the quantum light field when we cross-correlate $C_{11}$ with $C_{22}$ and $C_{23}$. We confirm strong photon antibunching without significant change of the light statistics compared to free-space emission (Fig. $4 \mathrm{c})$ and 4 e), respectively).

Finally, we evaluate the performance of the routing process by comparing the saturation behavior of the NV center emission into free space with its emission into the photonic structure. We obtain the net count rate by subtracting the background (linearly increasing with pump power) from the overall counts and fit according $\mathrm{tc}^{29}$ : $I(P)=\frac{\mathrm{I}_{\mathrm{Sat}}}{1+\mathrm{P}_{\mathrm{Sat}} / \mathrm{P}}$ where $\mathrm{I}_{\mathrm{Sat}}, \mathrm{P}_{\mathrm{Sat}}$ are the saturated count intensity and pump power, respectively. The free space emission of the $\mathrm{NV}$ center, obtained by adding $\mathrm{C}_{11}$ and $\mathrm{C}_{21}$, saturates at a count level of $(15 \pm 0.2) \cdot 10^{4} \mathrm{CPS}$ at a pump power of $(120 \pm 7) \mu \mathrm{W}$. This saturation level is significantly higher when compared to an NV center in bulk 11 which we attribute to a thin film effect ${ }^{32}$ combined with the NV center's polarization-dependent coupling to the ring. At the same time, the combined counts from the outcoupling gratings give $(15 \pm 0.1) \cdot 10^{3} \mathrm{CPS}$ at saturation at a pump power of $(100 \pm 4) \mu \mathrm{W}$. Assuming that the collection efficiency is the same as in the free-space case we could estimate an overall routing efficiency of $10 \%$. The simulations furthermore indicate a total collection efficiency of $\approx 15 \%$ at the gratings for a single photon emitted from the NV center if on resonance with the cavity mode. We note that reduced photon counts collected from gratings are largely due to the confocal nature of our experimental apparatus 
which collects light only from a small $\left(<1 \mu \mathrm{m}^{2}\right)$ region of the grating. The collection from the gratings could be significantly improved if light from the whole grating regions is collected using a multimode fiber or an objective lens. Improvements in the design of the gratings themselves can increase the collection efficiency up to $90 \% 33$. Finally, inverse-taper waveguide outcoupling $34 \mid 35$ could be used to efficiently collect most of the emitted light directly from the waveguide, without a need for a grating.

Our first demonstration of an integrated on-chip optical network based on diamond illustrates the great potential of a DOI platform in the field of quantum optics. The compact architecture and low loss material make our diamond platform suitable for large scale integration where multiple devices can be connected via single photon channels, thus enabling on-chip photonic networks. With the recent progress of spin-photon entanglement with single NV centers 36 our approach of providing photons by a single NV center placed in the resonator mode paves the way for integrated, scalable quantum networks 26 . In this case, single photons are used as transmitters of quantum information enabling fast, long distant information transfer to connect different nodes of the quantum network. Due to their long spin coherence times at room temperature, NV centers are ideal memory qubits to store quantum information. At the same time, the large Raman-gain, lack of multi-photon absorption processes at NIR and excellent thermal properties of diamond will likely lead towards the implementation of novel, high power, integrated active and passive networks based on our diamond platform. Examples include the realization of coherent light sources based on Raman scattering at wavelengths where semiconductor lasers are hard to implement, and nonlinear mixing processes that could lead towards wide-bandwidth comb generation $37 / 38$. Another future direction of our work is to enter the strong coupling regime. This can either be accomplished by improving the quality factor of the resonator or alternatively, by using photonic crystal cavities fabricated directly in diamond to achieve smaller mode volumes. Potential applications of strong light matter interaction include the realization of single photon transistors 39140 and quantum networks can be foreseen.

\section{METHODS}

Passive NIR measurements: Two tunable lasers (Santec, TSL 510) covering together a wavelength range from $1480 \mathrm{~nm}-1680 \mathrm{~nm}$ were used to obtain spectra at a sweep speed of $10 \mathrm{~nm} / \mathrm{s}$.

Two-collection arm confocal microscope: Measurements in the VIS were performed in a two-collection arm scanning confocal microscope where light is coupled at normal incidence to the device. A frequency doubled Nd:YAG laser (Coherent, Compass 315M-100) at $532 \mathrm{~nm}$ is used to pump and scan across the ring using a piezo-electrically controlled scanning stage. Several filters, a dichroic mirror reflecting green laser light and the collection fiber acting as a pinhole ensure that only light covering the range $600 \mathrm{~nm}-800 \mathrm{~nm}$ reaches the spectrometer or light in the range of $650 \mathrm{~nm}-800 \mathrm{~nm}$ is collected at two single photon counting modules (SPCM, Perkin Elmer, AQRH-14). The light that propagates to collection arm $\mathrm{C} 1$ is used to obtain a scan of the device. The pump beam is then placed at a fixed position and $\approx 50 \%$ of the emitted light is directed towards collection arm C2 via a beam splitter while a scanning mirror (SM, Newport) delivers a second confocal micrograph. The second scan reveals out-scattered light from light coupled to the device. For photoluminescence measurements we use grating spectrometers (HORIBA Jobin Yvon, iHR550 and Acton SpectraPro SP300i) where light is detected via a charged coupled device (CCD) detector. For all $g^{(2)}$-measurements no background subtraction was performed revealing a good contrast even in a type Ib diamond. This type of diamond usually has a high background level that is absent here due to the small amount of diamond material which shows that our diamond thinning process has led to removal of large amount of background originating from i.e. nearby NV centers, that would normally make such $g^{(2)}$-measurements difficult in the bulk.

Simulations: For a dipole (in-plane polarization) placed in the field maximum of a $5 \mu \mathrm{m}$ diameter ring resonator having a $250 \mathrm{~nm}$ x $250 \mathrm{~nm}$ cross-section we performed 3D FDTD simulations using the commercially available software Lumerical. To estimate the amount of light that we can collect from the waveguide gratings we monitor both grating ends. The distance between ring and waveguide is assumed to be $50 \mathrm{~nm} .15 \%$ of the overall dipole emission is routed to and upscattered at both grating ends combined for wavelengths on resonance. $30 \%$ of the overall dipole emission is scattered downwards at the gratings into the $\mathrm{SiO}_{2}$ layer where light can also get reflected back at the $\mathrm{SiO}_{2} / \mathrm{Si}$ interface and maximally $7 \%$ is transmitted through the grating. The mode volume of the ring resonator is obtained using

$$
V=\frac{\int \epsilon(r)|E(r)|^{2} d^{3} r}{\max \left(\epsilon(r)|E(r)|^{2}\right)} \approx 28 \cdot\left(\frac{\lambda}{n_{\text {diam }}}\right)^{3} .
$$

assuming that the dipole is placed in the field maximum with optimal polarization (aligned with the E-field of the cavity mode). Combining this value with the $\mathrm{Q}$ factor we measured we estimate a Purcell factor $\frac{30}{\sqrt{30}}$ of $F_{P}=\frac{3}{4 \pi^{2}} \cdot\left(\frac{\lambda}{n_{\text {diam }}}\right)^{3} \cdot \frac{Q}{V} \approx$ 8.7 . 


\section{CONTRIBUTIONS}

B. Hausmann, P. Maletinsky and J. Choy developed and carried out fabrication of devices. B. Hausmann, B. Shields and M. McCutcheon characterized the devices. Q. Quan accomplished all simulations. Data was analyzed by B. Hausmann and A. Kubanek. The paper was written by B. Hausmann, A. Kubanek and M. Lončar. All authors discussed the results and the content of the paper.

\section{ACKNOWLEDGEMENTS}

Devices were fabricated in the Center for Nanoscale Systems (CNS) at Harvard. The authors thank Parag Deotare for many helpful discussions and Daniel Twitchen from Element Six for support with diamond samples. T.M.B. was supported by the NDSEG and the NSF graduate student fellowships, and J.T.C. by the NSF graduate student fellowship. A.K. acknowledges support from the Alexander von Humboldt Foundation. This work was supported in part by Harvard's Nanoscale Science and Engineering Center (NSEC), NSF, DARPA QuEST program, and the Hewlett Packard Foundation as well as AFOSR MURI. M.L. acknowledges support from the Sloan Foundation.

\section{ADDITIONAL INFORMATION}

The authors declare that they have no competing financial interests. The views, opinions and/or findings contained in this publication are those of the author and should not be interpreted as representing the official views or policies, either expressed or implied, of the Defense Advanced Research Projects Agency or the Department of Defense. Correspondence and requests for materials should be addressed to M.L. (email: loncar@seas.harvard.edu)

1 Zaitsev, A. M. Optical properties of diamond: a data handbook. Springer-Verlag, (2001).

${ }^{2}$ Wrachtrup, J. and Jelezko, F. J. Phys.: Condens. Matter 18, 807-824 (2006).

${ }^{3}$ Neumann, P., Mizuochi, N., Rempp, F., Hemmer, P., Watanabe, H., Yamasaki, S., Jacques, V., Gaebel, T., Jelezko, F., and Wrachtrup, J. Science 320, 1326 (2008).

${ }^{4}$ O'Brien, J. L. Science 318, 1567 (2007).

${ }^{5}$ Maze, J. R., Stanwix, P. L., Hodges, J. S., Hong, S., Taylor, J. M., Cappellaro, P., Jiang, L., Dutt, M. V. G., Togan, E., Zibrov, A. S., Yacoby, A., Walsworth, R. L., and Lukin, M. D. Nature 455, 644 (2008).

${ }^{6}$ Balasubramanian, G., Chan, I. Y., Kolesov, R., Al-Hmoud, M., Tisler, J., Shin, C., Kim, C., Wojcik, A., Hemmer, P. R., Krueger, A., Hanke, T., Leitenstorfer, A., Bratschitsch, R., Jelezko, F., and Wrachtrup, J. Nature 455(7213), 648-651 October (2008).

7 Maletinsky, P., Hong, S., Grinolds, M., Hausmann, B., M.D.Lukin, Walsworth, R.-L., Loncar, M., and Yacoby, A. Submitted.

8 Childress, L., Taylor, J. M., Sørensen, A. S., and Lukin, M. D. Phys. Rev. Lett. 96, 070504 (2006).

9 Beveratos, A., Brouri, R., Gacoin, T., Villing, A., Poizat, J. P., and Grangier, P. Phys. Rev. Lett 89, 187901 (2002).

10 Dadap, J. I., Focht, G. B., Reitze, D. H., and Downer, M. C. Opt. Lett. 16 (7), 499-501 (1991).

11 Babinec, T., Hausmann, B. M., Khan, M., Zhang, Y., Maze, J., Hemmer, P. R., and Loncar, M. Nature Nanotech. 5, 195-199 (2010).

12 Hadden, J. P., Harrison, J. P., Stanley-Clarke, A. C., Marseglia, L., Ho, Y.-L. D., Patton, B. R., OŠBrien, J. L., and Rarity, J. G. Appl. Phys. Lett. 97, 241901 (2010).

13 Siyushev, P., Kaiser, F., Jacques, V., Gerhardt, I., Bischof, S., Fedder, H., Dodson, J., Markham, M., Twitchen, D., Jelezko, F., and Wrachtrup, J. Appl. Phys. Lett. 97, 241902 (2010).

14 Schröder, T., Gädeke, F., Banholzer, M. J., and Benson, O. New J. Phys. 13, 055017 (2011).

${ }^{15}$ Choy, J. T., Hausmann, B. J. M., Babinec, T. M., Bulu, I., Khan, M., Maletinsky, P., Yacoby, A., and Loncar, M. Submitted.

16 Barclay, P. E., Fu, K.-M. C., Santori, C., and Beausoleil, R. G. Appl. Phy. Lett. 95, 191115 (2009).

17 Wang, C. F., Choi, Y.-S., Lee, J. C., Hu, E. L., Yang, J., and Butler, J. E. Appl. Phy. Lett. 90, 081110 (2007).

18 Wang, C. F., Hanson, R., Awschalom, D. D., and Hu, E. L. Appl. Phy. Lett. 91, 201112 (2007).

19 Hiscocks, M. P., Ganesan, K., Gibson, B. C., Huntington, S. T., Ladouceur, F., and Prawer, S. Opt. Express 16 (24), 19512-19519 (2009).

${ }^{20}$ Loncar, M., Babinec, T., Choy, J., Hausmann, B., Bulu, I., Zhang, Y., Khan, M., and McCutcheon, M. W. In Artificial Atoms in Diamond: From Quantum Physics to Applications, (2010).

${ }^{21}$ Hausmann, B., Choy, J., Quan, Q., McCutcheon, M., Maletinsky, P., Babinec, T., Chu, Y., Kubanek, A., Yacoby, A., Lukin, M., and Loncar, M. In CLEO/QELS 2011, (2011).

22 Faraon, A., Barclay, P. E., Santori, C., Fu, K.-M. C., and Beausoleil, R. G. Nature Photon. 5, 301-305 (2010).

${ }^{23}$ Cai, M., Painter, O., and Vahala, K. J. Phys. Rev. Lett. 85 (1), 74-77 (2000). 
${ }^{24}$ Chao, C.-Y. and Guo, L. J. Appl. Phys. Lett. 83 (8), 1527 (2003).

25 Akimov, A. V., Mukherjee, A., Yu, C. L., Chang, D. E., Zibrov, A. S., Hemmer, P. R., Park, H., and Lukin, M. D. Nature 450, 402-406 (2007).

26 Kimble, H. J. Nature 453, 1023-1030 (2008).

27 Brown, R. H. and Twiss, R. Q. Nature 177, 27-29 (1956).

${ }^{28}$ Kimble, H. J., Dagenais, M., and Mandel, L. Phys. Rev. Lett. 39, 691 (1977).

${ }^{29}$ Kurtsiefer, C., Mayer, S., Zarda, P., and Weinfurter, H. Phys. Rev. Lett. 85, 290 (2000).

30 Purcell, E. M. Phys. Rev. 69, 681 (1946).

31 Collins, A. T., Thomaz, M. F., and Jorge, M. I. B. J. Phys. C 16, 2177 (1983).

${ }^{32}$ Lee, K. G., Chen, X. W., Eghlidi, H., Kukura, P., Lettow, R., Renn, A., Sandoghdar, V., and Goetzinger, S. Nature Photon. 5, 166 (2011).

33 Laere, F. V., Roelkens, G., Ayre, M., Schrauwen, J., Taillaert, D., Thourhout, D. V., Krauss, T. F., and Baets, R. J. Lightwave Technol. 25 (1), 151-156 (2007).

34 Almeida, V. R., Panepucci, R. R., and Lipson, M. Opt. Lett. 28 (15), 1302-1304 (2003).

35 Shoji, T., Tsuchizawa, T., Watanabe, T., Yamada, K., and Morita, H. Electron. Lett. 38 (25), 1669-1670 (2002).

36 Togan, E., Chu, Y., Trifonov, A. S., Jiang, L., Maze, J., Childress, L., Dutt, M. V. G., Sørensen, A. S., Hemmer, P. R., Zibrov, A. S., and Lukin, M. D. Nature 466, 730-734 (2010).

37 Zhi, M., Wang, X., and Sokolov, A. V. Opt. Exp. 16 (16), 12139 (2008).

38 Del'Haye, P., Schliesser, A., Arcizet, O., Wilken, T., Holzwarth, R., and Kippenberg, T. J. Nature 450, 1214-1217 (2007).

39 Chang, D. E., Sørensen, A. S., Demler, E. A., and Lukin, M. D. Nat. Phys. 3, 807-812 (2007).

${ }^{40}$ Hwang, J., Pototschnig, M., Lettow, R., Zumofen, G., Renna, A., Götzinger, S., and Sandoghdar, V. Nature 460, 76-80 (2009).

(a)

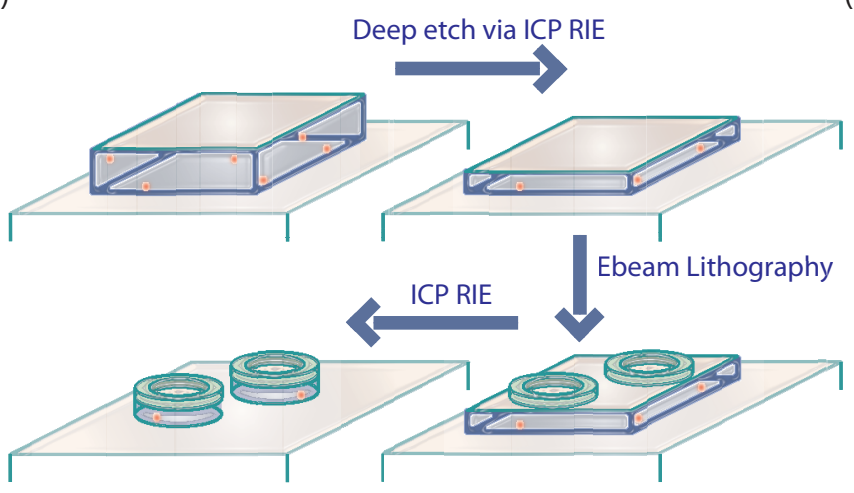

(b)

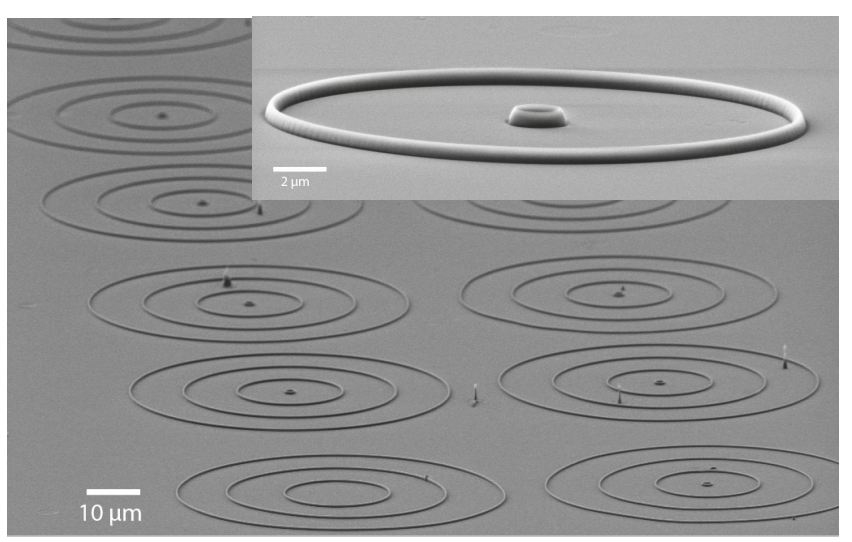

FIG. 1: Single crystal diamond-on-insulator platform. (a) Fabrication schematic: We thin a diamond slab via an oxygen based ICP RIE, spin a layer of spin-on-glass e-beam resist, use e-beam lithography to define a ring shaped mask, and transfer the mask through the thinned diamond using ICP RIE. Residual resist stays on devices during characterization. Optically

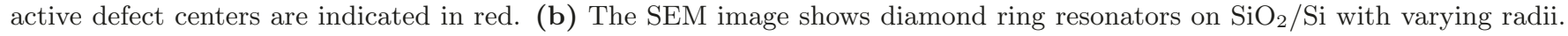
Inset: Higher magnification image of two ring resonators with smooth sidewalls. 
(a)

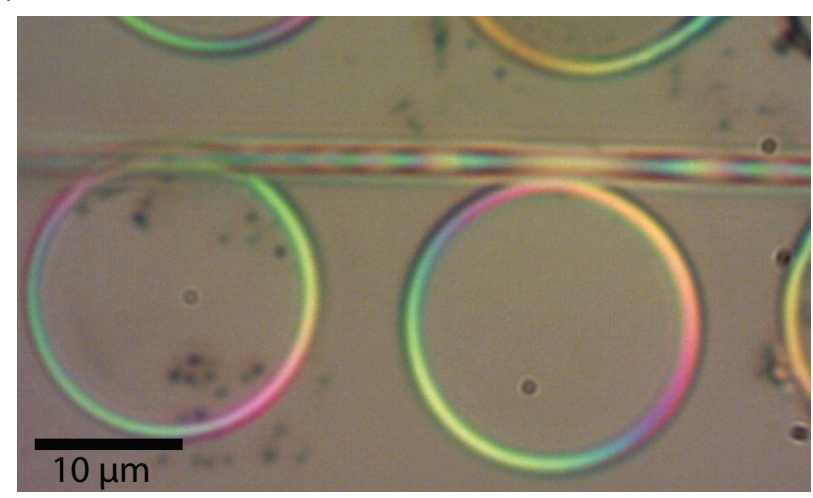

(c)

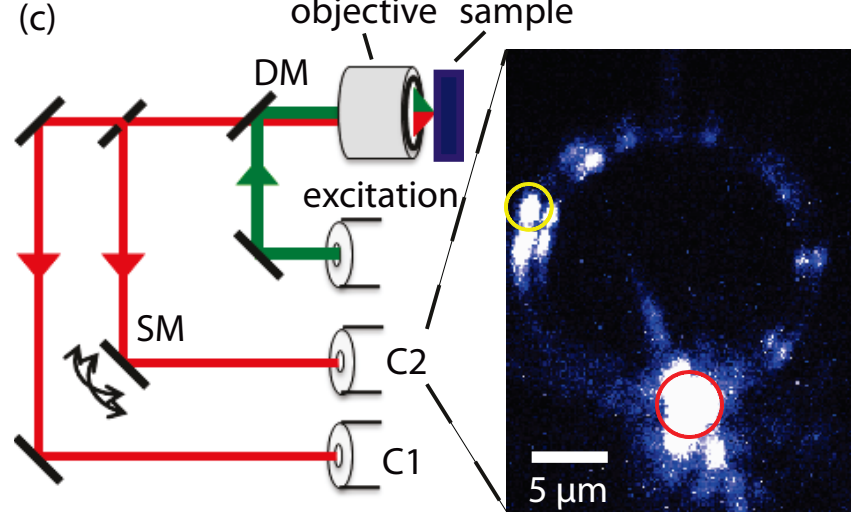

(b)

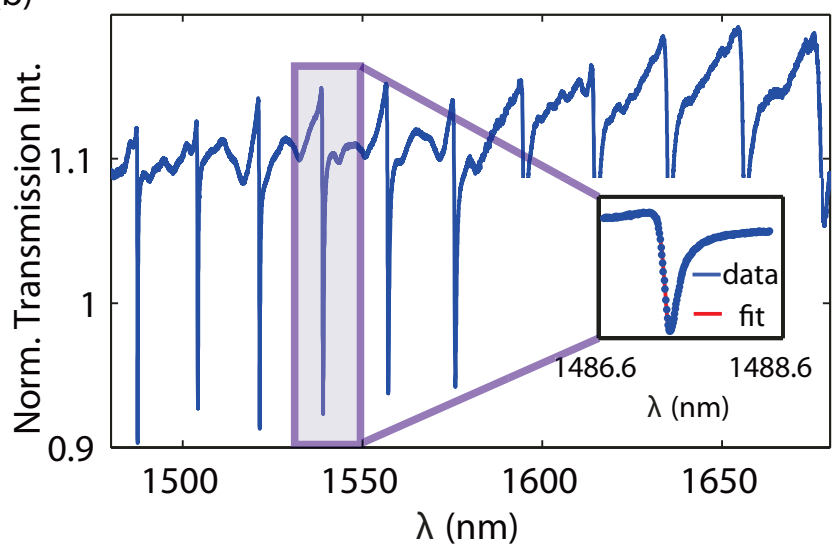

(d)

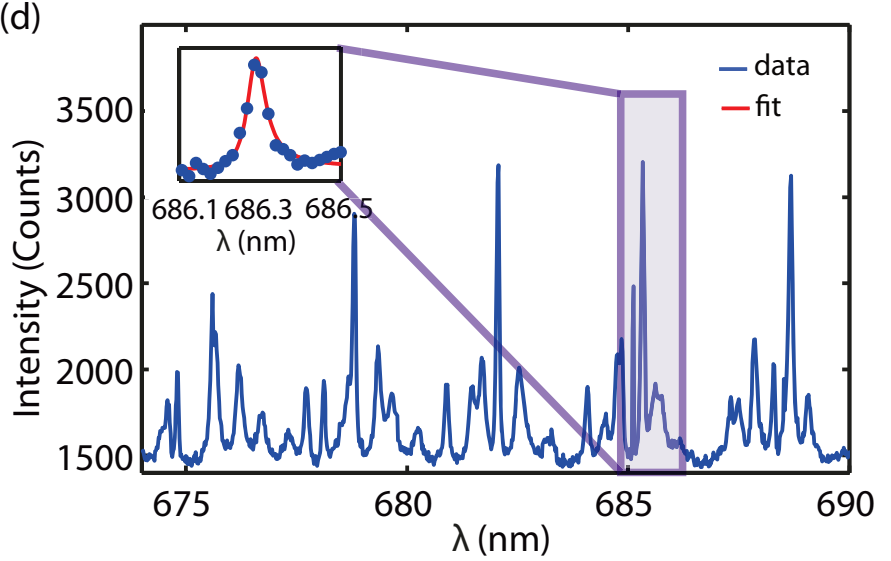

FIG. 2: Broadband high-Q performance of single ring resonators. (a) Optical image shows single ring resonators (20 $\mu \mathrm{m}$ diameter, $410 \mathrm{~nm}$ thick and covered with $300 \mathrm{~nm}$ thick low-index resist layer) on a quartz substrate while a tapered fiber is approached to the ring in the center of the image. (b) Transmission measurements unveil periodic Fano-like modes over a wavelength range of $1480-1680 \mathrm{~nm}$ in a tapered fiber set-up. The $\mathrm{Q}$ factors obtained via Fano-like fits range from $\approx 700$ $(1678 \mathrm{~nm})$ to $\approx 9000(1487 \mathrm{~nm})$. Inset shows blow-up of the transmission dip at $1487 \mathrm{~nm}$. (c) Schematic of a two collection arm confocal microscope used in the VIS characterization of diamond ring resonators (See text for details). After scanning the stage where the sample is mounted on the pump beam (and C1) is located at the indicated spot (red) and a second scan is performed using collection arm 2 (C2) via a scanning mirror (SM) (c, right). The yellow circle marks the collection position while taking spectra. (d) The photoluminescence spectrum contains periodic whispering gallery modes. A pump power of $1.5 \mathrm{~mW}$ is used at an integration time of $300 \mathrm{~s}$. Inset: A Q-factor of $(12.6 \pm 1) \cdot 10^{3}$ was obtained by a Fano-like fit (red) of a representative mode. 
(a)

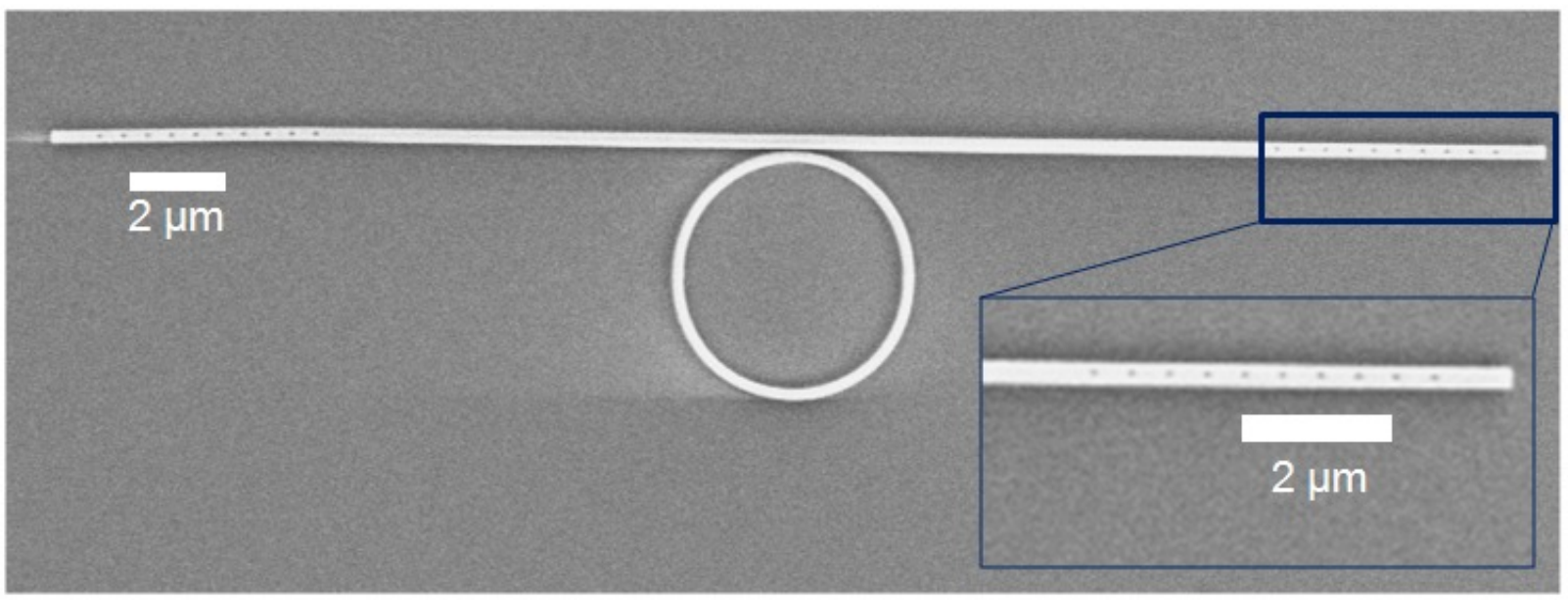

(b)

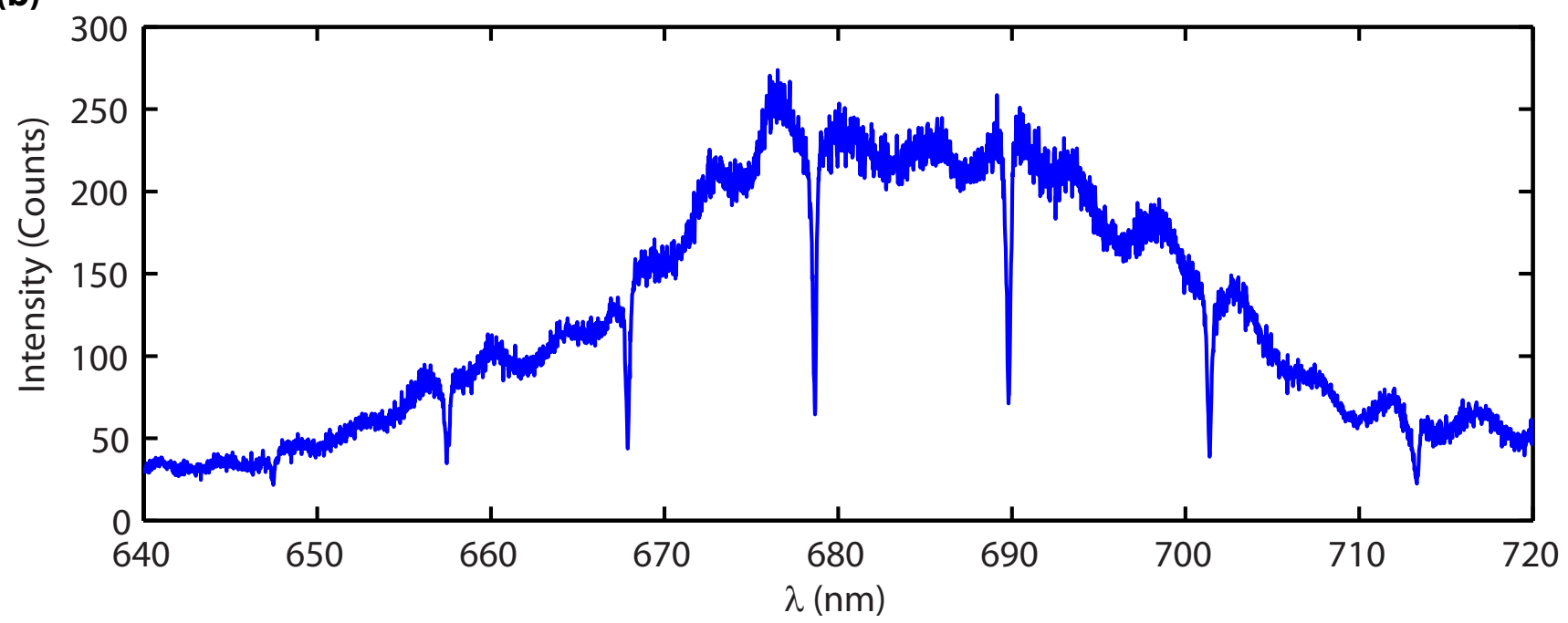

FIG. 3: Realization of an all-diamond photonic network on chip: Waveguide integrated ring resonator platform. (a) SEM image of a single mode ring resonator coupled to a waveguide containing second order gratings on both ends which facilitate collection of single photons via upward scattering. The ring diameter is $5 \mu \mathrm{m}$ in the presented case, having a $245 \mathrm{~nm}$ ring width. The gap between the waveguide and the ring is $100 \mathrm{~nm}$, while the waveguide itself has a width of $370 \mathrm{~nm}$. The devices are sitting on $\mathrm{SiO}_{2} / \mathrm{Si}$ substrate. Inset: Higher magnification image of the grating region. (b) Transmission spectrum through the waveguide via white light excitation exhibits a periodic mode structure. 
(a)

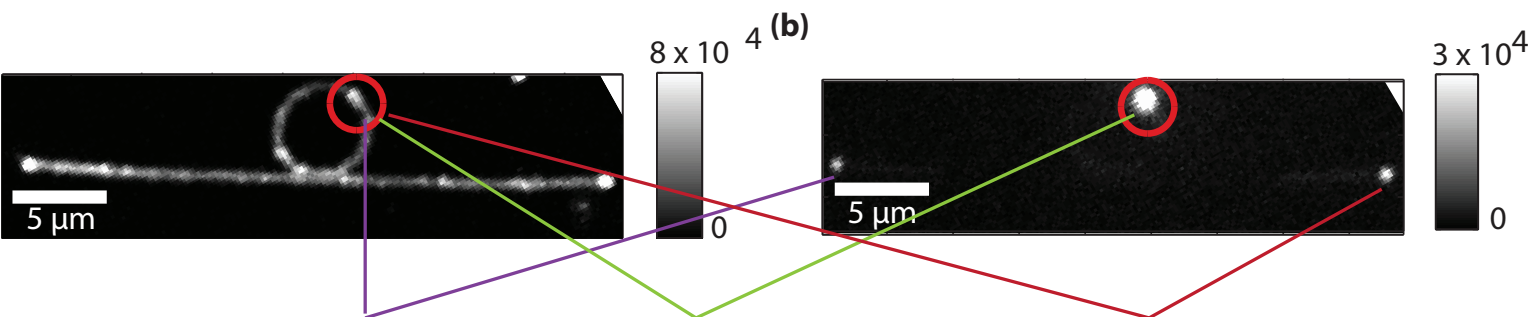

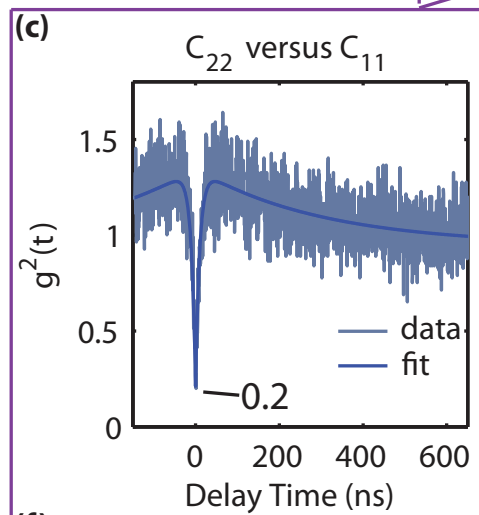

(f)
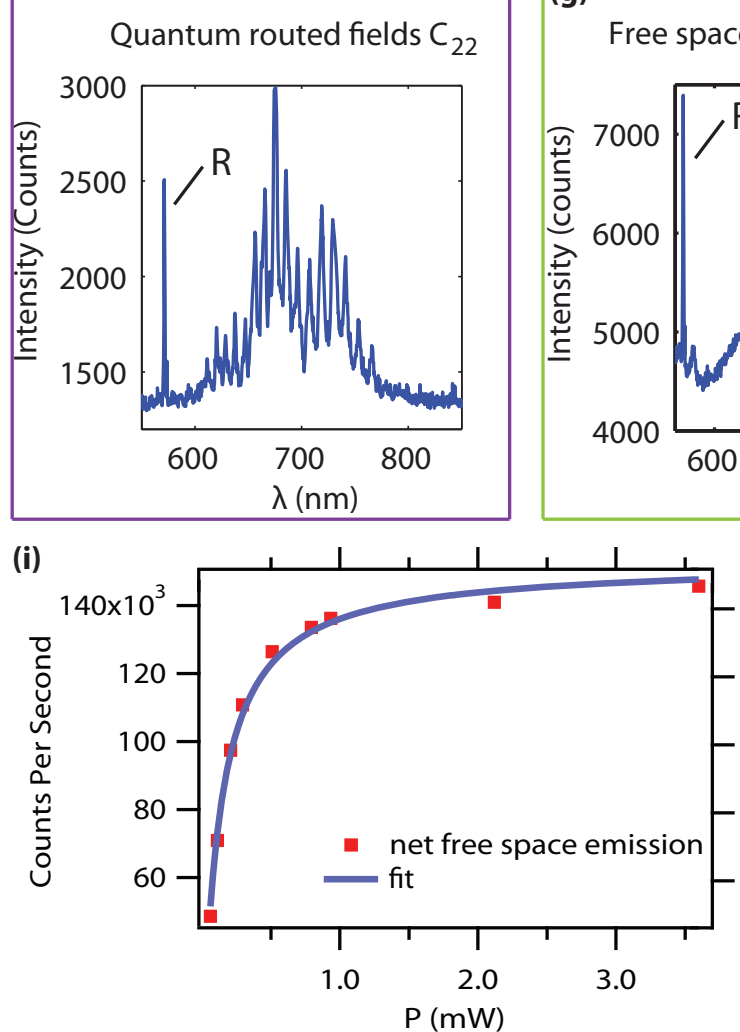

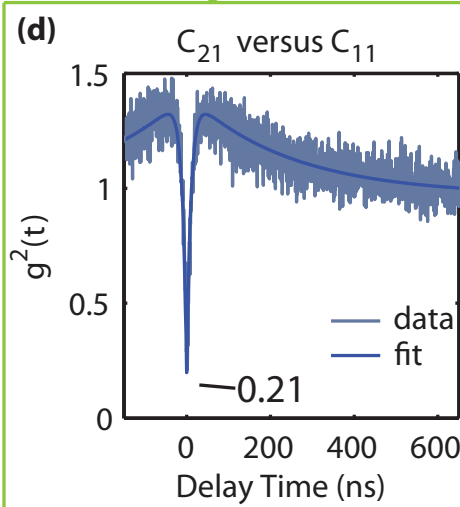

(g)

Free space emission $C_{11}+C_{21}$

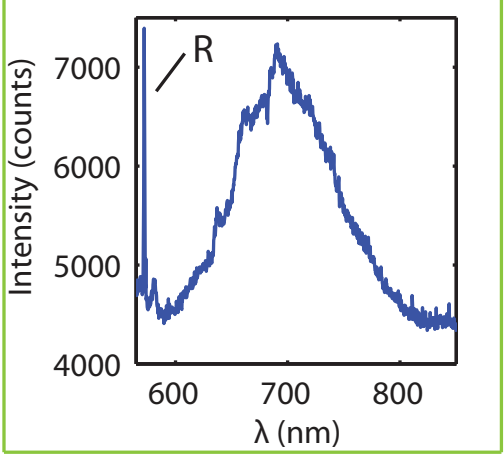

(k)

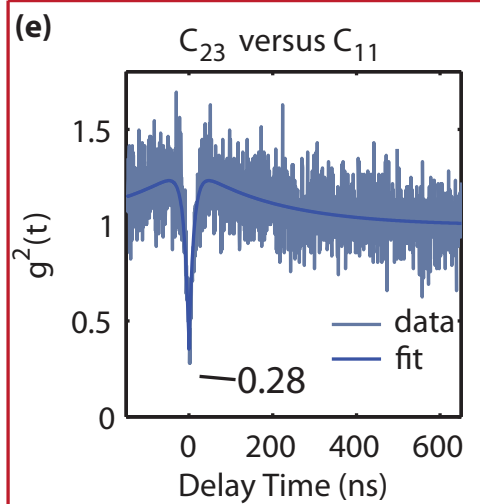

(h)

Quantum routed fields $\mathrm{C}_{23}$
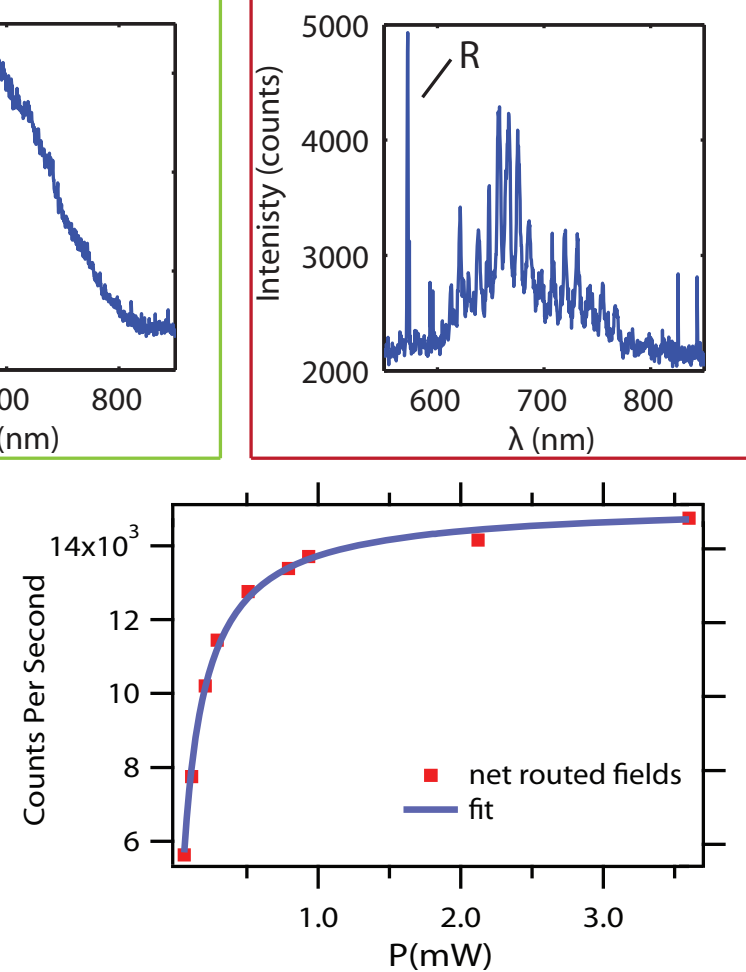

FIG. 4: Single photon generation and routing in diamond networks. (a) We obtain a confocal scan over our routing device in $\mathrm{C} 1$. (b) We fix the pump beam at the NV center (collection position $\mathrm{C}_{11}$ ) and a scan of collection arm 2 shows three collection positions: $\mathrm{C}_{21}$ for free-space collection from the $\mathrm{NV}$ center, and at both gratings $\left(\mathrm{C}_{22}, \mathrm{C}_{23}\right)$. (c-e) We compare the collection at $\mathrm{C}_{11}$ with collection at $\mathrm{C}_{21}$ as well as both ends of the waveguide $\left(\mathrm{C}_{22}, \mathrm{C}_{23}\right)$. Autocorrelation measurements reveal nonclassical light emission in three scenarios: Correlating the free-space coupled single photon flux from collection channel $\mathrm{C}_{11}$ versus $\mathrm{C}_{21}$, (d), and correlating $\mathrm{C}_{11}$ versus the routed collection positions $\mathrm{C}_{22}$ and $\mathrm{C}_{23}$, (c) and (e) respectively. The pump power is $200 \mu \mathrm{W}$ and we perform the measurements for $400 \mathrm{~s}, 800 \mathrm{~s}$ and $2400 \mathrm{~s}$ respectively. We observe a good contrast in all cases $\left(g^{(2)}(0)<0.5\right.$, no background subtraction has been performed). (g) The spectrum shows the characteristic emission from an NV center that we obtain by combining collection $\mathrm{C}_{11}$ and $\mathrm{C}_{21}$. The exact same position of the (non-broadened) Raman line at $573 \mathrm{~nm}$ as in the bulk diamond indicates that the single crystal diamond film quality is comparable to bulk diamond (denoted by R). (f), (h) Spectra collected from the gratings $\mathrm{C}_{22}, \mathrm{C}_{23}$, respectively, reveal whispering gallery modes superimposed on the NV center emission spectrum with a 150 lines $/ \mathrm{mm}$ grating. We obtain a Q-value of $(3.2 \pm 0.4) \cdot 10^{3}$ for $665.9 \mathrm{~nm}$ with a high resolution grating. (i) Free-space collection exhibits a saturated single photon flux of $(15 \pm 0.2) \cdot 10^{4} \mathrm{CPS}$ at a pump power of $120 \pm 7 \mu \mathrm{W}$ from an NV center. The net counts from a single NV center are obtained via subtracting the linear background from the overall count rate. (k) The combined count rate at both gratings gives a saturation level of $(15 \pm 0.1) \cdot 10^{3} \mathrm{CPS}$ at a saturation pump power of $(100 \pm 4) \mu \mathrm{W}$. 